\title{
CONSIDERACIONES EN TORNO AL DIAGNÓSTICO PREIMPLANTATORIO A PROPÓSITO DEL CASO CAMILA ${ }^{1}$
}

\author{
Carla Navarro Fernández ${ }^{2}$
}

\begin{abstract}
RESUMEN
A fines del año 2010, una pareja de esposos anunció que demandaría a una clínica limeña, ya que, tras haberse sometido a una fecundación in vitro, la madre alumbró mellizas, siendo que una de ellas, Camila, nació con Síndrome de Down, afecciones cardiacas congénitas e hipertensión pulmonar, condiciones que, según aquella, "la clínica pudo evitar si hacía un análisis genético antes de implantar los embriones".

¿Acaso Camila era un producto fallado? Algunos utilitaristas sustentarían estas palabras; sin embargo, desde la bioética personalista, el análisis es muy distinto, ya que se parte de la noción de la sacralidad de la vida humana. A fin de que el Perú no se convierta en una sociedad que renuncie a la búsqueda de relaciones justas y simétricas entre iguales racionales con los mismos derechos y deberes, es al derecho a quien le corresponde ejercer un control sobre ciertas prácticas científicas que ponen en peligro los principios democráticos de los Estados modernos. Así, la vida de Camila cobrará un nuevo sentido.
\end{abstract}

\section{PALABRAS CLAVE}

Diagnóstico preimplantatorio. Dignidad humana. Derecho a la vida. Cosificación del embrión. Personalismo.

\begin{abstract}
At the end of 2010, a couple announced that it would file charges against a private hospital in Lima because, after undergoing in vitro fertilization, the mother gave birth to twins, one of which, Camila, was born with Down Syndrome, congenital heart conditions, and pulmonary hypertension. According to the mother, such conditions "could have been prevented by the hospital if it had performed a preimplantation genetic diagnosis of embryos before they were transferred to the uterus."

Was Camila a damaged product? Some utilitarians would believe so; however, from the Personalist Bioethics perspective, the analysis is quite different since the starting point is the sanctity of human life. In order to prevent Peru from becoming a society giving up on its quest for fair and symmetric relations among equals who enjoy the same rights and duties, Law is called upon to limit certain scientific practices that threaten the democratic principles of modern States. Hence, Camila's life will have a new meaning.
\end{abstract}

Key Words

Preimplantation diagnosis. Human Dignity. Right to Life. Objectification of Embryos. Personalism.

\section{INTRODUCCIÓN}

A fines del año 2010, una noticia en nuestro país impactó a propios y extraños: una pareja de esposos anunció que demandaría en Lima a la Clínica Concebir por daños y perjuicios y por negligencia médica. Tras haberse sometido a una fecundación in vitro, la madre alumbró a mellizas, siendo que una de ellas, Camila, nació con Síndrome de Down, afecciones cardiacas congénitas e hipertensión pulmonar, condiciones que, según aquella, "la clínica pudo evitar si hacía un análisis genético antes de implantar los embriones" .
El caso suscitó polémica debido a que se mencionaron las palabras "estafa" y "producto fallado" en un contexto que poco tendría que ver con la finalidad última de la procreación: dar vida sin jugar a ser Dios en el ínterin.

En este sentido, lo que pretende el presente trabajo es anali-

1 El verdadero nombre de la protagonista se ha cambiado a fin de respetar sus derechos fundamentales que podrían verse vulnerados en caso de mencionar su identidad.

2 Estudiante del XII ciclo de Derecho de la Universidad Femenina del Sagrado Corazón - UNIFE.

3 Padre de bebe in vitro: “¿Cómo se sentiría si le dieran un producto fallado?”, 2010, en http://elcomercio.pe/lima/667840/ noticia-padre-bebe-in-vitro-como-se-sentiria-si-le-dieran-producto-fallado (consultado el 24 de julio del 2012). 
zar, a partir del caso relatado, en qué consiste el procedimiento de diagnóstico preimplantatorio y si debería aplicarse o no en este tipo de casos, enfatizando el contexto científico en el que surge esta técnica, para luego resaltar algunas consideraciones bioéticas a partir de la trasgresión del imperativo Kantiano y de las advertencias de Habermas ${ }^{4}$, permitiendo de este modo motivar al lector a cuestionarse sobre la tendencia al posible descontrol de las tecnologías en nuestra sociedad.

\section{DiAGNÓSTICO PREIMPLANTATORIO}

En primer lugar, debemos conocer en qué consiste esta técnica para luego profundizar sobre los aspectos ético jurídicos de la misma.

\section{Aspectos científicos}

Los padres de Camila señalan que los médicos debieron detectar las anomalías genéticas en estado embrionario. En efecto, el diagnóstico preimplantatorio, al surgir en el contexto de las técnicas de reproducción asistida, plantea en su primera etapa la de diagnóstico, es decir: "microbiopsar al embrión de 72 horas y, mediante la obtención de una o dos células del mismo, someterlas al estudio citogenético o molecular indicados". ${ }^{5}$
Sin embargo, al ser una ciencia que mide sus resultados en términos de eficiencia y eficacia, se incrementó la investigación con embriones humanos, lo que supone que éstos sufran una cierta modificación en cuanto a su valoración, puesto que, además de ser objetos de producción, también lo son de experimentación. Tal situación incrementa así su carácter de cosa, es decir, el control de calidad del producto y es aquí donde retumban las palabras de los demandantes al señalar que la clínica les había prometido los mejores embriones, los más vivos, los más capacitados.

Nos sometemos así a las leyes de la clásica eugenesia negati$v a$, donde la selección se realiza por descarte del producto defectuoso. Sin perjuicio de ello, cabe resaltar que ahora se habla de una eugenesia positiva, por lo que ahora también se elige que sobreviva a través del diagnóstico aquel embrión portador de una determinada carga genética. ${ }^{6}$

En síntesis, la dinámica de producción y consumo de la Fecundación in Vitro se ha ido perfeccionando del siguiente modo: a) en cuanto al método, que ha llevado a formas de fecundación tecnificadas, b) en relación al control del producto, con la eliminación de aque- llos mal hechos y c) con una producción de calidad a través de la selección de embriones a transferir?.

Ahora bien, ¿qué sucede con los embriones que no han sido escogidos? Los médicos de la clínica Concebir expresan que si los padres hubieran aceptado el diagnóstico genético preimplantacional, (ya que ellos aducen que sí les informaron del proceso y que se negaron) y rechazaran a la niña, el compromiso era congelar el embrión.

Cabe aquí resaltar que en el Perú no hay protocolos médicos ni leyes que regulen qué destino corresponde a los embriones fecundados in vitro que no son implantados en el vientre materno. Es por ello que las instituciones médicas, como la mencionada clínica, siguen los parámetros de la Red Latinoamericana de Reproducción Asistida que recomienda la implantación de no más de tres embriones fecundados, así como la preservación (congelamiento) en caso de los no implantados.

\section{Aspectos Bioéticos}

Tras analizar brevemente en qué consiste en Diagnóstico preimplantatorio podemos preguntarnos cuál es la justificación ética de los partidarios de esta práctica, para luego develar la

4 Jürgen Habermas (Düsseldorf, 18 de junio de 1929) es un filósofo y sociólogo alemán, conocido sobre todo por sus trabajos en filosofía práctica (ética, filosofía política y del derecho). Gracias a una actividad regular como profesor en universidades extranjeras, especialmente en Estados Unidos, así como por la traducción de sus trabajos más importantes a más de treinta idiomas, sus teorías son conocidas, estudiadas y discutidas en el mundo entero.

5 Cano, M., Diagnóstico Preimplantatorio, Buenos Aires, Facultad de Ciencias Jurídicas de la Universidad del Salvador, 2006, p. 2

6 Con el caso Nash se ha dado el primer paso hacia la denominada construcción de embriones y por primera vez se ha manipulado un embrión para beneficio de un tercero existente.

7 PASTOR, L., "Diagnóstico Genético Preimplantatorio", en Mujer y realidad del aborto: un enfoque interdisciplinar. Asociación Extremeña de Amigos del Foro Español de la Familia, 2008, p. 3. 
interrogante de si nacer (aún con la posibilidad de desarrollar una enfermedad que afecte la calidad de vida) es mejor que no nacer.

\section{¿Derecho a tener hijos?}

Los padres de Camila consideran que tener hijos es un derecho. Bajo esta perspectiva, parte de este derecho es que sean sanos. Más aún, si han invertido dinero en que les "hagan" hijos tienen derecho a reclamar si el producto tiene algún defecto. Ello concordaría con la opinión de ciertos entendidos del tema, los cuales exclaman que:

“También el DGP8 puede configurarse como un elemento valioso para el desarrollo de la libertad reproductiva de aquellos padres que desean garantizar las mejores condiciones posibles de salud para sus hijos. Se habla entonces del surgimiento de un nuevo concepto de paternidad responsable que se vale de la información genética para decidir sus opciones reproductivas y de un nuevo tipo de eugenesia, la denominada 'neoeugenesia' que presenta matices propios importantes que la diferencian de forma clara de la eugenesia clásica (esencialmente el hecho de que tiene su origen en la libertad reproductiva de los individuos o de las parejas, y no en políticas estatales como antaño)."9
Esta visión mercantilista de la procreación se integra perfectamente con la concepción de las técnicas de fecundación in vitro, siendo que éstas se encuentran en el ámbito de los procesos productivos $\mathrm{y}$, como tales, sujetas a las leyes del mercado. Es por ello que no es exagerado señalar que "el embrión humano in Vitro está condicionado por las tendencias de la oferta y la demanda y sometido al criterio de calidad del producto"10.

\section{El estatuto bioético del em- brión in vitro}

En primer lugar, hay que tener en cuenta que la preferencia de la técnica del diagnóstico preimplantatorio respecto al aborto como mecanismo para evitar el nacimiento de descendientes enfermos, se apoya en la hipótesis de que los embriones jóvenes tienen menor rango moral que los fetos e, incluso, en el convencimiento para algunos de que los embriones in vitro con alguna tara genética carecen totalmente de valor ético ${ }^{11}$.

Entre los exponentes más destacados de la nueva separación entre las nociones de "persona y ser humano" tenemos a $\mathrm{H}$. Tristam Engelhardt y a Peter Singer. El primero considera que el fundamento del respeto debido a las personas es su autonomía moral, siendo que no tiene sentido hablar de respeto de los embriones ya que aún no son au- tónomos o nunca lo fueron. Por su parte, para Singer, la noción de persona no está ligada a la especie humana ya que el valor de las vidas humanas es variable entre otras cosas porque la vida sin autoconciencia no tiene ningún valor. ${ }^{12}$

Podemos encauzar dichos argumentos dentro de la esfera del utilitarismo, teoría de la bioética que sostiene que el resultado de una acción, para todos aquellos que serán afectados por ella, determina si esa acción es moralmente correcta o no. Se realiza un cálculo costo-beneficio y el resultado práctico es lo que se decide. Con estas bases, la bioética utilitarista sustentaría los siguientes conceptos ${ }^{13}$ :

$>$ El bien se identifica con el bienestar del mayor número de personas.

$>$ El fin puede justificar los medios, en orden a conseguir este resultado beneficioso.

$>$ La vida humana tiene un valor relativo y extrínseco, lo que significa que tendrá el valor que consideramos que tiene.

$>$ Enfatiza el valor de la calidad de la vida, por encima del valor de la vida en sí, que valdrá sólo en función de su calidad.

Las palabras de la madre de Camila son contundentes: "si hubiera sabido que mi hija tendría estos problemas, no la habría

\footnotetext{
Siglas para Diagnóstico Genético Preimplantacional. Abellan, F., “Aspectos bioéticos y legales del diagnóstico genético preimplantatorio (DGP)”, en Revista de la Escuela de
Medicina Legal, 2006, vol. 23 (núm. 2) , p. 15 .

10 Pastor, L., 2008, p. 236.

11 Abellán, F., 2006, p. 126.

12 Andorno, R., “¿Todos los seres humanos son personas?”, en XVI Jornadas Nacionales de Derecho Civil, 1997, Comisión n9: Bioética y Derecho Civil.

13 López, M. y Abellán, J., Los Códigos de la Vida, Madrid, Homolegens, 2009. p, 103.
} 
tenido"14, es decir, para ella, el diagnóstico preimplantatorio supone una especie de práctica abortiva precoz y no está lejos de la realidad. En efecto, como hemos visto, tal técnica con el tiempo ha devenido en la expresión de aquella mentalidad eugenésica que acepta el aborto selectivo para impedir el nacimiento de niños afectados por varios tipos de anomalías. Así, los olvidados son los embriones descartados, que son considerados como un estorbo para los padres.

\section{La dignidad humana y el impe- rativo Kantiano}

Desde la bioética personalista, el análisis es muy distinto, ya que se parte de la noción de la sacralidad de la vida humana y de la convicción de que cualquier acto sobre el hombre y su corporalidad debe ser respetuoso de su dignidad personal. Así, los rasgos más importantes de esta corriente serían los siguientes ${ }^{15}$ :

- El bien, lo correcto éticamente, pende directamente de la verdad. Por lo tanto, una acción en relación con la persona será bioéticamente lícita si es conforme con las exigencias derivadas de esa naturaleza personal.

- El ser humano, que es siempre persona, ha de ser tratado siempre como un fin en sí mismo, nunca como un medio.

$>$ La vida humana posee un valor intrínseco, es inviola- ble e intangible. A esto (y no a ningún aspecto religioso) se refieren los personalistas cuando hablan de la sacralidad de la vida humana.

$>$ El progreso científico y tecnológico está subordinado al bien de la persona humana.

Desde esta perspectiva, quienes consideran al embrión in vitro como realidad personal, señalan que éste debe protegerse como persona desde que el óvulo ha sido fecundado. Según esta tesis, el embrión humano, desde el momento de la concepción, tiene un status moral equivalente al de una persona, que le hace acreedor al pleno respeto de su integridad física.

Si manipulamos genéticamente un embrión, estaríamos ante un caso claro de transgresión del denominado imperativo categórico Kantiano, en su tercera formulación, que de acuerdo con el propio Kant dice así: "Obra de tal modo que uses la humanidad, tanto en tu persona como en la persona de cualquier otro, siempre como un fin al mismo tiempo y nunca como un medio"16

Es decir, para Kant es fundamental que se reconozca que los seres racionales son fines en sí mismos y nunca sólo medios de otros. Entonces, si aplicamos estas reflexiones teóricas de Kant a la situación concreta de Camila, podríamos decir que, al declarar sus padres que tenían muchas ilusiones de tener un bebe, que invirtieron tiempo $y$ dinero y viajes para ahora ver a su hija sufrir, se está utilizando a la niña como un medio para satisfacer la necesidad de otras personas como si aquella fuese de su propiedad y no tuviera una existencia propia y autónoma.

\section{Normatividad vigente ¿incons- titucionalidad?}

El preámbulo de la Convención sobre los Derecho Humanos y la Biomedicina ${ }^{17}$, del Consejo de Europa, establece "la necesidad de respetar al ser humano, no sólo como individuo, sino también en su pertenencia a la especie humana" reconociendo la importancia de garantizar su dignidad. En el mismo sentido, el artículo 2, apartado a) de la Declaración Universal sobre el Genoma Humano y los Derecho Humanos ${ }^{18}$, mantiene que "cada individuo tiene derecho al respeto de su dignidad y derechos, cualesquiera que sean sus características genéticas". En su punto b) afirma que "esta dignidad impone que no se reduzca a los individuos a sus características genéticas y que se respete su carácter único y su diversidad".

En el Perú, la Carta Magna protege la vida desde su concepción, es decir, nuestro sistema jurídico es vitalista y antiabortista. En esta misma línea, el Tribunal Constitucional señala que la concepción de un nuevo ser humano se produce con la fusión de las células materna y paterna con lo cual se da ori-

14 Padre de bebe in vitro: “¿Cómo se sentiría si le dieran un producto fallado?”, 2010.

15 López M. y Abellán, J., 2009, pp. 105-107.

16 Kant, E., Fundamentación metafísica de las costumbres, México, Pomia, 1996. p. 44.

Convenio Europeo sobre los Derechos Humanos y la Biomedicina, 4 de abril de 1997.

18 Declaración Universal sobre el Genoma Humano y los Derechos Humanos, 11 de noviembre de 1997. 
gen a una nueva célula que, de acuerdo al estado actual de la ciencia, constituye el inicio de la vida de un nuevo ser. ${ }^{19}$

Además, el concebido es sujeto de derecho en todo cuanto le favorece, ello sin contar con los dispositivos legales acordes con el texto constitucional, tales como el Código Civil, el Código de los Niños y Adolescentes y la Ley General de Salud. Sin embargo, al consagrar ésta última en su artículo 7 el uso de las técnicas de reproducción asistida, la fecundación in vitro estaría permitida. Frente a ello surge la siguiente controversia: si, en la práctica, tal y como hemos analizado, el objeto de un diagnóstico preimplantacional ha devenido en deshacerse de un embrión con anomalías y dicha técnica es un paso más en el procedimiento de la fecundación in vitro, resulta urgente el cuestionar la constitucionalidad del artículo 7 de dicho cuerpo normativo, pues al practicarse estas técnicas bajo este esquema, se atentaría contra la vida y dignidad del concebido; además, el Tribunal Constitucional se pronuncia protegiendo al ser humano desde el momento de la concepción.

LAS ADVERTENCIAS DE HABERMAS Y ALGUNAS REFLEXIONES PERSONALES

Resulta importante culminar el presente trabajo analizando las argumentaciones filosóficas que hizo Jürgen Habermas acerca del diagnóstico preimplantatorio $^{20}$, que han influido en la decisión actual de Alemania y de otros países de la Comunidad Europea de poner límites legales al uso indiscriminado del procedimiento en sus sociedades. En suma, el pensamiento de dicho autor se basa en las siguientes ideas:

Aceptar que hoy vivimos en sociedades pluralistas, donde cada individuo puede tener distintas concepciones de lo que es la vida buena. Sin embargo, también cree que se podría establecer un consenso social si se acepta que lo que se llama "convivencia moral" de alguna manera tiene que ver con una convivencia justa.

$>$ Cuando el DGP se propone para una eugenesia positiva que beneficie a terceros, 0 dependa de lo que los padres crean que es mejor para sus hijos, entonces esa manipulación biológica sobre el embrión lo lleva a perder su inviolabilidad moral, que depende de su indisponibilidad orgánica.

De lo anterior se concluye la necesidad que considera Jürgen Habermas en garantizar el derecho de los seres humanos a poseer una herencia genética no manipulada en su estado embrionario.

En síntesis, si una sociedad acepta y no regula la manipulación eugenésica sobre los embriones humanos por medio, en este caso del DGP, será una comunidad que ha renunciado a la búsqueda de relaciones justas y simétricas entre iguales racio- nales con los mismos derechos y deberes. Al parecer, tal situación es la del Estado Peruano, ya que, como según se ha podido analizar, por un lado es una sociedad que, muy a pesar de lo que redacta la Constitución en cuanto a la protección a la vida desde su concepción, acepta las técnicas de fecundación asistida sin una reglamentación necesaria; por otro lado no hay protocolos médicos ni leyes que regulen qué destino corresponde a los embriones fecundados in vitro que no son implantados en el vientre materno. Tamaña paradoja no hace sino empeorar las cosas, puesto que lo único que se logra es romperse la igualdad entre los humanos, siendo ello un grave factor desequilibrador de la paz social.

Es por ello que considero crucial que la tendencia al descontrol de las tecnologías que se mencionó al inicio del presente trabajo sea amortizada con el reconocimiento intrínseco que el derecho de la ciencia al progreso y de la sociedad a alcanzar avances que mejoren nuestra calidad de vida, no puede derivar en la cosificación del ser humano. Es al derecho a quien le corresponde ejercer un control sobre ciertas prácticas científicas que ponen en peligro los principios democráticos de los Estados modernos, a fin de que la vida de Camila cobre un nuevo sentido y nosotros progresemos como nación. En definitiva, comparto la opinión de Aparisi quien señala que " $e l$ respeto a la dignidad humana genera que, en el ámbito cien-

19 STC del 16 de Octubre del 2009. (Expediente número 2005-2009-PA/TC). Gaceta Constitucional, № 28, Gaceta Jurídica, Lima, 2010.

20 En diciembre del año 2001 publicó un texto en alemán, que fue traducido en el 2002 al español con el título de "El futuro de la naturaleza humana ¿Hacia una eugenesia liberal?. 
tífico, no todo lo que se puede hacer debe hacerse"21.

\section{Conclusiones}

La demanda de una pareja de esposos contra una clínica de fertilidad por el nacimiento de Camila, una niña con Síndrome de Down, ha despertado polémica sobre las técnicas de fecundación asistida en el Perú, en las que se observa una evolución (o tal vez involución) de no sólo dar un hijo a quien naturalmente no puede tenerlo, sino que lo que ahora se persigue es dar un hijo de buena calidad.

Uno de los baluartes de esta nueva medicina es el diagnóstico genético preimplantatorio, el cual consiste en una técnica diagnóstica para comprobar la "salud genética" de un embrión, antes de decidir su transferencia al útero materno o su descarte para una finalidad procreativa. Es decir, parte de una consideración utilitarista de la vida siendo que el embrión no puede ser catalogado como persona puesto que no tiene autonomía moral. Tendencia contraria es la personalista, para la cual toda vida ha de ser protegida y defendida. Así lo estipulan tanto declaraciones internacionales como nuestra Constitución. Ello, sin embargo, no es del todo claro, puesto que en leyes sectoriales se ha normado la fertilización in vitro sin una adecuada legislación, quedando desprotegido jurídicamente el embrión en su derecho a la vida.

Es por ello que se requieren debates fructíferos, amplios y democráticos, tomando referencia la legislación comparada, a fin de que se logren consensos entre la población, desde una perspectiva humanista y se decida cómo deben ser regulados estos nuevos avances tecnológicos, para evitar crear algo que inevitablemente acabe destruyendo nuestra dignidad humana.

\section{Biblografía}

ABELLAN, Fernando. "Aspectos bioéticos y legales del diagnóstico genético preimplantatorio (DGP)". En: Revista de la Escuela de Medicina Legal. Vol. 23- $\mathrm{n}^{\circ} 2$. Madrid, Setiembre de 2006.

ANDORNO, Roberto. "¿Todos los seres humanos son personas?". En XVI Jornadas Nacionales de Derecho Civil. Comisión n9: Bioética y Derecho Civil. Buenos Aires, setiembre de 1997.

APARISI M, Angela. "Selección genética de embriones y dignidad humana" Publicado en Diario Noticias (Pamplona), el 19 de marzo de 2002.
CANO, María E. "Diagnóstico Preimplantatorio". Artículo publicado por la Facultad de Ciencias Jurídicas de la Universidad del Salvador de Argentina el 15 de mayo del 2006.

Convenio Europeo sobre los derechos humanos y la biomedicina, 4 de abril de 1997.

Declaración Universal sobre el Genoma Humano y los Derechos Humanos, 11 de noviembre de 1997.

KANT, E. "Fundamentación metafísica de las costumbres" México, editorial Pomia. 1996.

LOPEZ. B, Mónica y ABELLAN, José." Los Códigos de la Vida". Madrid. Homolegens, 2009.

PASTOR GARCIA, Luis. “Diagnóstico Genético Preimplantatorio". En: Mujer y realidad del aborto: un enfoque interdisciplinar. Asociación Extremeña de Amigos del Foro Español de la Familia. Cáceres. 2008.

Sentencia del Tribunal Constitucional del 16 de Octubre del 2009. Gaceta Constitucional, № 28, Gaceta Jurídica, Lima, 2010.

21 Aparisi, A. “Selección genética de embriones y dignidad humana”, en Diario Noticias (Pamplona), el 19 de marzo de 2002. 